\title{
Pediatric clinical trials: current scenario in the Asia Pacific region
}

This article was published in the following Dove Press journal:

Pediatric Health, Medicine and Therapeutics

4 July 2013

Number of times this article has been viewed

\section{Lisa Marie Saldanha' \\ Saumya Nayak' \\ Adeline Sng' \\ Mei-Ling Long' \\ Elisabeth Schrader ${ }^{2}$ \\ Amanur Rahman ${ }^{3}$ \\ Elvira Zenaida Lansang' \\ Karen Wai' \\ Ken Lee ${ }^{4}$}

'Feasibility and Site Identification Asia, Quintiles East Asia Private Limited, Singapore; ${ }^{2} \mathrm{Q}$ uintiles Pediatric Center of Excellence, Durham, NC, USA; ${ }^{3}$ Faculty of Engineering, National University of Singapore, Singapore; ${ }^{4}$ Asia Site Services, Quintiles East Asia Private Limited, Singapore
Correspondence: Lisa Marie Saldanha Quintiles East Asia Private Limited, 79 Science Park Drive, \#06-08, Cintech IV, Science Park One, Singapore 118264

Tel +65 $66021000 /+6597246122$

Fax +65 68720430

Email lisamarie.saldanha@quintiles.com
Objective: This site survey was conducted to understand the current pediatric clinical trial landscape across countries in the Asia Pacific region, specifically in terms of interest, experience, capabilities, requirements of the ethics committee, patient availability, and overall challenges involved in conducting pediatric trials.

Methods and materials: Between May and June 2012, an English language survey form was sent to sites (identified through Quintiles' internal database) with pediatric capability and referrals from doctors during a preliminary outreach. In July 2012, the responses from the completed survey forms were entered into SurveyMethods, a web-based central repository. Data analysis was performed in August-September 2012 using SurveyMethods.

Results: Seventy-seven sites were contacted for this survey across the Asia Pacific region. Sixty-four percent (49 sites) completed 63 surveys and confirmed interest to participate in clinical trials in the pediatric population. Seventy-one percent of the sites had prior experience. Eighty percent confirmed needing an assent from pediatric patients; $81 \%-95 \%$ confirmed acceptance of placebo-controlled and pharmacokinetic studies by ethics committees; and $37 \%$ cited challenges in conducting studies in this population.

Conclusion: This survey indicates that there is a high level of interest among sites in the Asia Pacific region in conducting pediatric trials across various therapeutic indications. No major insurmountable challenges were identified in conducting pediatric trials. Complexity of the consent and assent process across the countries needs to be considered. Also to be considered are the sites' needs as well as trial design adaptations for this population to ensure compliance (minimize invasive procedures, placebo control, visit schedules versus school timings, etc). There also appears to be an adequate patient pool in many indications, and the availability of a database of healthy subjects can assist in faster screening of subjects for vaccine trials.

Keywords: Site survey, ethical, challenges, consent patients

\section{Introduction}

Pediatric trials have received a lot of attention in recent years, largely due to the introduction of pediatric regulations by the European Medicines Agency (EMA) and the US Food and Drug Administration (FDA) ${ }^{1-4}$ and the disparity between the pediatric disease burden and the representation of the pediatric population in clinical trials. ${ }^{5}$ Traditionally, drugs have not been tested for efficacy and safety for use in the pediatric population. As a result, the use of unlicensed and off-label medicines in the pediatric population has been extensive.

According to 2007 data, in the European Union (EU), more than half of the medicines frequently used in children have only been studied in adults. ${ }^{4}$ Also, an analysis on clinical trials conducted for selected conditions, representing a high burden of pediatric 
disease listed on ClinicalTrials.gov from 2006 to 2011, found that $59.9 \%$ of the disease burden for these conditions was attributable to children, but only $12.0 \%$ of trials were pediatric. This once again showed a significant discrepancy between the pediatric burden of disease and the amount of clinical trial research dedicated to pediatric populations. ${ }^{5}$

Both the US and EU legislators have made pediatric clinical trials a permanent component of the drug development process. Similarly, Asia Pacific countries are also following suit, with the Japanese Ministry of Health, Welfare, and Labor leading the way. ${ }^{6}$ To meet these regulatory requirements, the logical progression is for companies to conduct multicenter and multinational studies. In 2010, an analysis performed on published studies conducted under the US Pediatric Exclusivity Provision, which provides economic incentives to pharmaceutical companies to conduct clinical trials in children, found that $65 \%$ of the studies were conducted in one or more countries outside the US, and 11\% did not include any US sites. Also, $38 \%$ of trials enrolled one or more patients in developing countries. ${ }^{7}$ To ensure that adequate numbers of pediatric subjects are enrolled in a trial during a given time frame and to address the need to test therapies in the populations where they may be used while ensuring that the trials are financially viable, it is apparent that these trials will be expanding further into the developing regions. Against this backdrop, Quintiles initiated a survey of pediatric clinical trial sites to understand the current scenario in the Asia Pacific region.

This paper provides the results of the pediatric site survey and gives an overview of the current scenario in the Asia Pacific region for the conduct of pediatric clinical trials.

\section{Materials and methods}

The Quintiles pediatric survey in the Asia Pacific region was conducted as follows:

Between May and June 2012, an English-language survey form was sent to sites (identified through Quintiles' internal database) with pediatric capability and referrals from doctors during a preliminary outreach. The survey forms were designed by the Quintiles regional feasibility team. They were sent by email or posted directly to pediatricians at sites for which we had contact details; if not, they were sent to the clinical research centers, with instructions to forward the survey form to the appropriate pediatricians, according to the therapeutic areas covered in the survey. The survey covered questions on:

- pediatricians' interest in conducting pediatric clinical trials;

- the ethics committee (EC) requirements in the countries;
- challenges in conducting pediatric clinical trials;

- current site capability in terms of availability of experienced investigators and site staff, electronic data capture (EDC) experience, Good Clinical Practice (GCP), and other training;

- pediatric patient pool in five therapeutic areas (respiratory, oncology, endocrinology, cardiovascular, and central nervous system) and availability of a subject database (ie, a record of children who have been vaccinated at the site and could potentially be considered for a vaccine study).

The survey questions were a combination of closed and open-ended questions to allow quantitative and qualitative results. The closed questions required "yes" or "no" type responses and number value responses; eg, number of subjects seen per site per month per therapeutic area. The open-ended questions were included to address items such as challenges faced and documentation used. The survey form was also reviewed for content by a team of Quintiles' representatives with expertise in feasibility, medical, and project management aspects of clinical trials in pediatric patients.

In July 2012, the responses from the completed survey forms were entered into SurveyMethods, a web-based central repository. ${ }^{8}$ Appropriate follow-ups were conducted to encourage the return of completed surveys. Data analysis was performed in August-September 2012 using SurveyMethods (Figure 1).

\section{Results}

Seventy-seven sites were contacted for this survey across the Asia Pacific region. Forty-nine sites (64\%) completed the survey, and all confirmed interest to participate in clinical trials in the pediatric population within the survey time frame. The 142 pediatricians with various subspecialties were contacted from these 77 sites. Given the range of questions addressed, multiple pediatricians at a given site completed the survey for the site.

Only in India, two surveys were completed for each of the 14 sites contacted (ie, 28 surveys completed by 14 sites in India), bringing the total number of surveys received to 63 . Information from the two surveys at each of the sites in India was different; hence, each survey was considered unique.

\section{Discussion}

As stated by the World Health Organization: "Children are not little adults." "They represent a unique population, 
differing in distinct developmental and physiological aspects from adults. These fundamental aspects, combined with the need for age-appropriate outcome measures, family involvement in decision making, and adaptations of research processes to fit the various age groups, have made conducting clinical trials in the pediatric population challenging. They require careful execution to meet the expectations of patients, EC, regulatory authorities, and the pharmaceutical companies. ${ }^{10}$

The pediatric survey we conducted had a response rate and an interest rate of $64 \%$ among sites contacted (Figure 2). A $64 \%$ interest level can be explained by an increasing trend in the number of trials conducted in the pediatric population over the last few years in this region. In Australia and New Zealand, sites would not complete a questionnaire that was not related to a specific protocol due to time constraints. Most of the sites did say that if there was a feasibility survey about a specific protocol, they would review this and determine interest. Hence, while the response rate is low, this does not mean that the interest level is low. An analysis of the US ClinicalTrials.gov registry between July 2007 and September 2010 showed an increase in pediatric trials in north, east, and south Asia, with 38, 78, and 85 trials, respectively, being conducted from July 2005 to September 2007; 43, 135, and 100 trials were conducted from October 2007 to September 2010 in these subregions. ${ }^{11}$

\section{Ethical considerations}

The International Conference on Harmonisation (ICH)'s Efficacy Guideline 11 (E11), Clinical Investigation of Medicinal Products in the Pediatric Population, is one of a number of technical requirements for registration of pharmaceuticals for human use. Guideline E11 reminds us that conducting clinical trials in the pediatric population is essential and should be done without compromising the well-being of pediatric patients participating in clinical studies. This responsibility is shared by companies, RA, health professionals, and society as a whole. ${ }^{12}$ Keeping this in mind, one of the survey's objectives was to understand better the local EC requirements and site capabilities to take on pediatric trials across the Asia Pacific countries. Table 1 shows the responses provided by the survey. The use of a placebo arm in clinical trials always raises a debate and much more so for trials in this vulnerable population. ${ }^{13,14}$ The results indicate a general acceptance of placebo-controlled trials (81\%) and trials involving pharmacokinetic (PK) analysis (95\%) by ECs in the region. However, these points were also raised as potential challenges by most sites (Figure 3). To help ECs make an informed decision, respondents suggested the provision of a strong justification for the use of a placebo. Methods to manage placebo in pediatric trials have been widely discussed. Potential strategies include: excluding high-risk patients, minimizing the number of patients on placebo, providing rescue medication, and maximizing safety assessments. ${ }^{15,16}$

Respondents have suggested that study assessments (including PK sampling) should concentrate on minimizing discomfort, pain, and fear of invasive procedures. Additionally, younger children often have needle phobia, making obtaining the blood sample also a challenge. Globally, regulators have discussed the use of population PK studies instead of the traditional design, as this involves more patients but fewer samples per patient, as a potential option for more acceptable PK studies in children. ${ }^{17}$ Employing the right study staff is also another important aspect when addressing the challenge of taking blood samples, which is discussed in more detail under Operational considerations.

\section{Consent considerations}

Another important consideration for pediatric trials is the consent process. This process often adds a lot of pressure to parents as they will be consenting for their child to participate in an experimental drug trial and may also subject them to invasive procedures. ${ }^{18}$ To first determine who could consent on behalf of a minor, an open-ended, free text question was included in the survey to understand if there were any local regulations/guidelines to define who a legally acceptable representative (LAR) was and also to understand better the practice being followed at sites. Survey respondents stated that

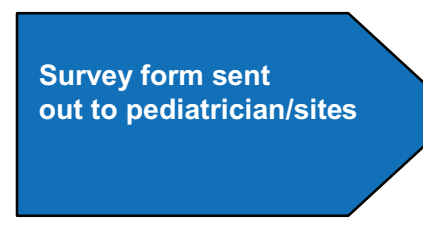

May-June 2012
Completed survey responses entered into a web-based central repository (survey methods)
Data analysis performed using survey methods

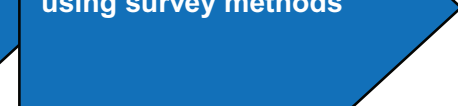

August-September 2012

Figure I Survey process and timeline. 


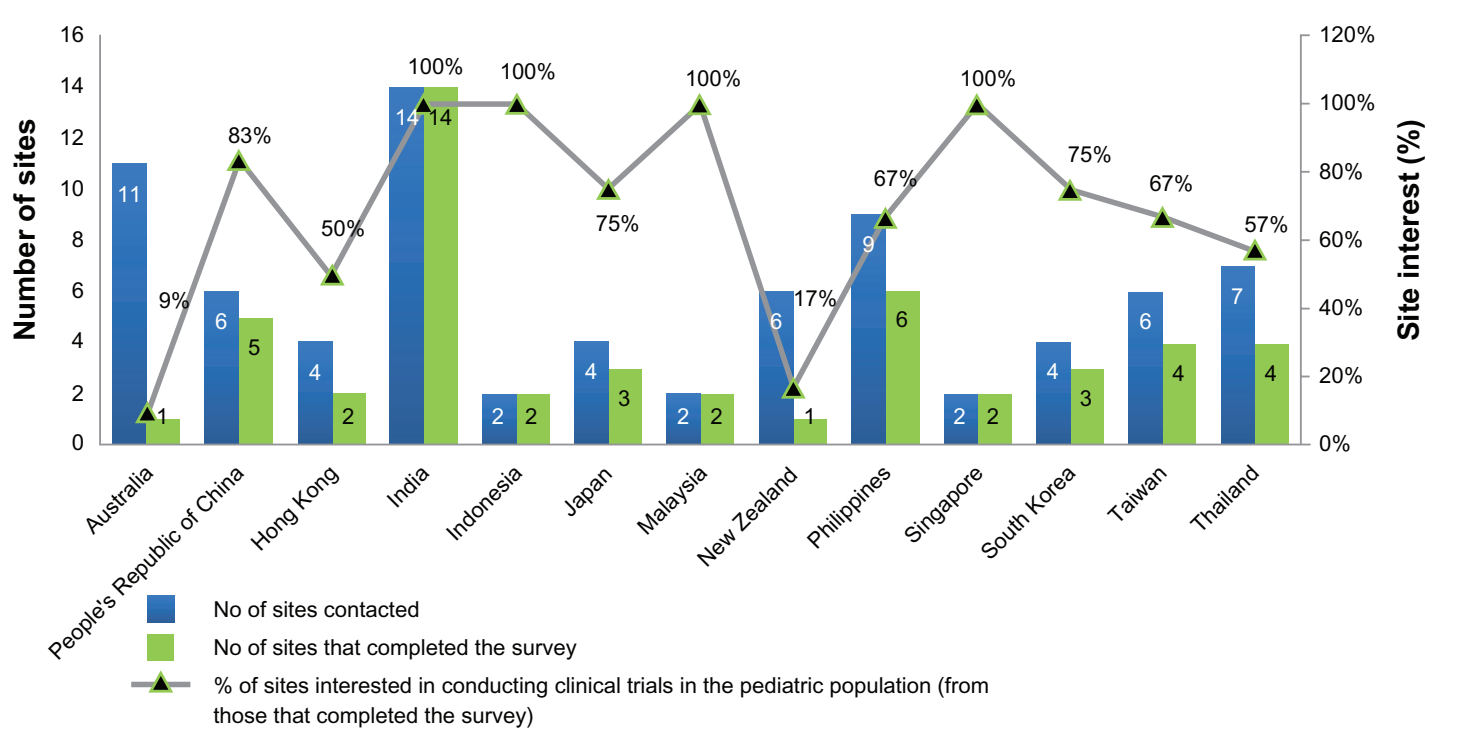

Figure 2 Number of sites that responded to the survey and the interest level of survey respondents to conduct clinical trials in the pediatric population per country.

parents are generally accepted (59\%) as the LAR to consent on behalf of children, and guardians can stand in as LARs when parents are deceased. Based on the survey results, only Japan requires both the parents to sign the consent. This requirement may also depend on the marital status of the parents. In Asia Pacific, availability of proof of relationship to the child is also important in some countries. Government-issued identification cards for parents and child and the child's birth certificate being the most popular and widely accepted documentation as highlighted by the respondents in Table 2 .

Table I Subspecialties of the 142 pediatricians who responded to the survey in Asia Pacific

\begin{tabular}{ll}
\hline Investigator subspecialty & Total number* \\
\hline Internal medicine & 16 \\
Cardiology & 18 \\
Pulmonology & 19 \\
Endocrinology & 19 \\
Nephrology & 17 \\
Rheumatology & 15 \\
Oncology & 20 \\
Hematology & 22 \\
Infectious disease & 23 \\
Neurology & 20 \\
Psychiatry & 14 \\
Surgery & 17 \\
Obstetrics and gynecology & 12 \\
Orthopedics & 16 \\
Radiology & 13 \\
Dermatology & 13 \\
Otolaryngology & 14 \\
Ophthalmology & 14 \\
Neonatal specialist & 24 \\
\hline Note: Pedain were
\end{tabular}

Note: *Pediatricians were allowed to select multiple specialties.
The patient's assent is another important requirement of the consenting process. In addition to the informed consent that is given by the LAR, all participants should be informed to the best possible extent about the study in language and terms they are able to understand. ${ }^{11}$ The assent form is designed to ensure that the child of appropriate intellectual maturity understands the potential risks and benefits of the trial and personally signs his assent. Eighty percent of survey respondents indicated that an assent is required, and they have provided a wide range of minimum age at which child assent is to be obtained. None of the countries showed consistency in the minimum age requirement, indicating that this is determined at a site level. This is in line with global findings. Feedback from a survey on 188 Institutional Review Boards (IRB) in the US found that most IRBs suggest a minimum age of 7 for a child to assent to participation in a clinical trial; however, the practice varies across sites, depending on the practice actually followed by the investigator. ${ }^{19}$ ICH E11 guidelines also state that the age of assent can be determined by the EC/IRBs or be consistent with local legal requirements. ${ }^{11}$ The American Academy of Pediatric Guidelines for the Ethical Conduct of Studies to Evaluate Drugs in Pediatric recommends assent for all children above the age of $7 .{ }^{20}$

The consent process has been raised as a challenge by survey respondents in many countries (Figure 3 ) and will need special consideration in planning and execution. One respondent from the People's Republic of China cited that the One Child Policy ${ }^{21}$ can influence consent, requiring additional time to discuss with parents. (In general, the One Child Policy in People's Republic of China is a policy by 


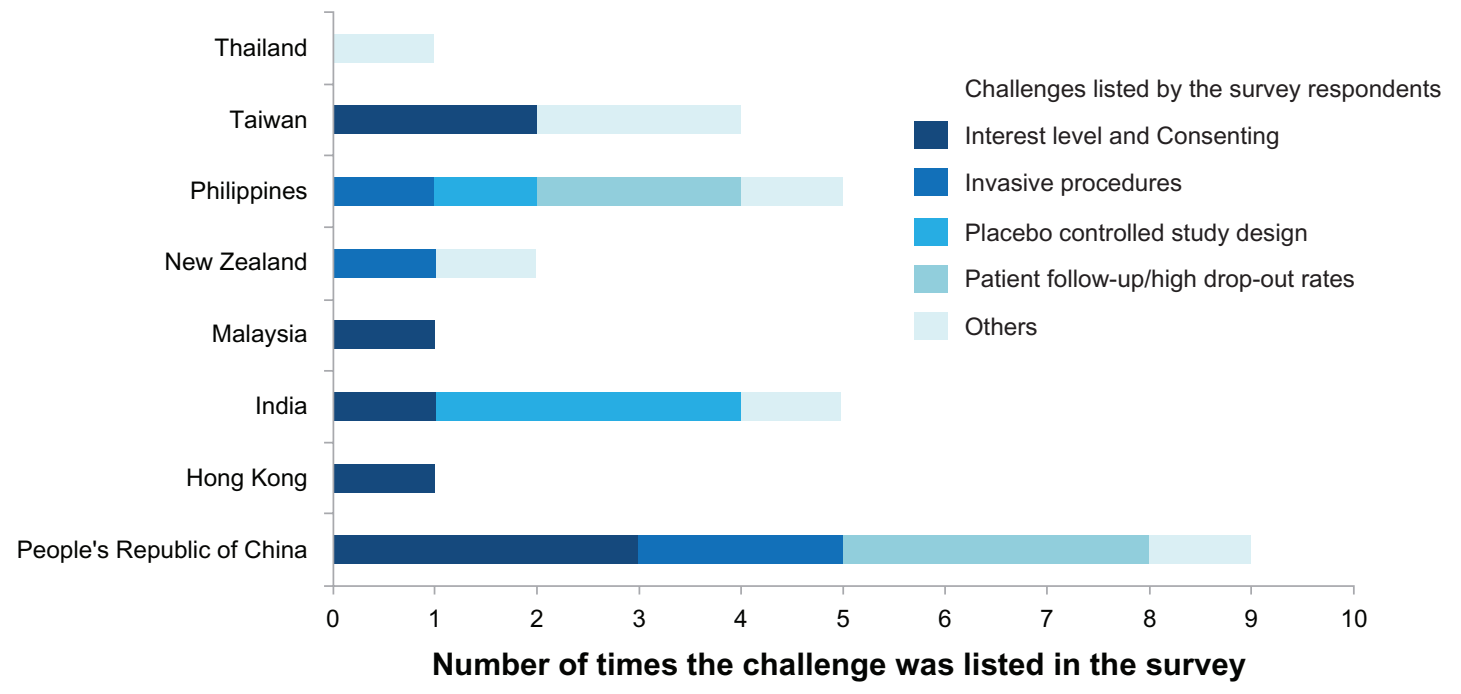

Figure 3 Overview of the survey response to the types of challenges faced in conducting clinical trials in the pediatric population.

the government to control population growth. It restricts the family to bear only one child. The details of this policy are beyond the remit of this paper). A respondent from the Philippines mentioned that trials involving invasive procedures and placebo control further complicate the consenting process. According to Barfield and Church, the site staff should have a clear understanding of the risks and benefits of a trial and have ways to communicate with various age groups within the pediatric population to address the consent issue. ${ }^{22}$ The creative use of technology like animated assent forms, audiovisual, and multimedia informed consent programs can also aid the consent process. ${ }^{23}$ Overall, the process for obtaining parental consent for a child to participate in a clinical trial varies to some extent within the region as well as within a country.

\section{Continuity considerations}

Patient follow-up and a high dropout rate were other challenges mentioned by the respondents. This is also cited in ICH E11 section 2.5.5; noncompliance is a special problem in adolescents, particularly when medicinal products (for example, steroids) affect appearance. ${ }^{12}$ It is also known that privacy is of high concern in children aged 12-18 years, and care must be taken while discussing matters of contraception and birth control during a trial.

To enhance compliance, study visits should be designed to accommodate school schedules, as well as meal and sleep times, particularly for younger children, and also the work schedule of their parents/guardians. ${ }^{24}$ The formulation of the drug is also important in pediatric trials, with tablets and capsules less suitable for younger children. Even the flavor of the formulation can be an influencing factor. This is also emphasized in ICH E1 1 section 2.2 (pediatric formulations) and section 2.4.1 PK. ${ }^{12}$ Retention strategies, such as regular follow-ups, weekly phone calls, reimbursement of travel expenses, and patient/parent education, could be useful, according to the survey respondents.

The challenges listed above confirm that those reviewing pediatric research protocols must have adequate expertise in different areas of child health and research. It is also critical to adhere to a high ethical standard and to avoid any coercion, or appearance of coercion. ${ }^{25}$

\section{Operational considerations Availability of suitable resources and facilities}

Seventy-one percent of the survey respondents had experience in conducting global/local clinical trials in pediatric population, and $84 \%$ are GCP certified. Eightyone percent indicated experience using EDC, and $82 \%$ confirmed having study staff available for a pediatric trial, showing the region's experience and readiness to take on pediatric trials. A New Zealand respondent stated that in the case of most studies his site has conducted, the pediatric study nurse(s) have been the most critical to the success of recruitment and maintenance of children throughout the trial, highlighting the importance and acknowledgement of study staff in the trial. For successful pediatric trials, modifications in procedures, equipment, staffing, family communication, and other aspects of care that are tailored to their special developmental needs are essential. When the clinical trial staff members performing blood draws from a child have 
Table 2 Responses to questions on EC requirements, overall challenges faced, site capabilities, and patient databases in Asia Pacific

\begin{tabular}{lll}
\hline Questions raised to the 63 survey respondents* & $\begin{array}{l}\text { \% of respondents that } \\
\text { responded "yes" }\end{array}$ & $\begin{array}{l}\text { Minimum age range } \\
\text { indicated by respondents }\end{array}$ \\
\hline
\end{tabular}

\section{Ethics considerations}

Does your EC allow studies with a placebo arm

$81 \%$

to be conducted in the pediatric population?

Does the EC allow studies requiring blood sample

collection for PK analysis in the pediatric population?

Who is considered a legally acceptable representative

according to local regulations?

Is any documentation/ proof required for an individual

to be accepted as a legally acceptable representative?

Is this documentation readily available?

Is an assent required from pediatric patients?

If yes, what is the age above which an assent is required?

\section{Challenges}

Do you foresee any challenges with conducting pediatric trials at your site?

Site experience and capabilities

Have you conducted any global/local clinical trials in pediatric patients? $\quad 71 \%$

Are you Good Clinical Practice (GCP) certified?

Do you have experience in using EDC ${ }^{d}$ or electronic case report forms?

Are there clinical staff in your hospital who have adequate time to take

on the role of a study coordinator?

Patient databases

Does your site have a large database of healthy infants

$95 \%$

( $0-2$ years) and children (2-10 years)?

Does your site have a large database of adolescent subjects (10-19 years)? $\quad 70 \%$

Can the database be mined by age group and patient vaccine history? $\quad 68 \%$

Who is the primary point of contact for pediatric patients?**

$\mathrm{GP}=15 \%$

Usually at what age do patients move from a pediatrician to general

Parents/legal guardians $=32 \%$

Only parents $=27 \%$

$17 \%=$ No response

$24 \%=$ others $^{\mathrm{a}}$

$39 \%$ b

$67 \%$

$80 \%$

$5-16$ years

practitioners/specialists at your site (and in country as a whole)?

Notes: *Some respondents have not answered all questions; **some respondents in India, the Philippines, and Malaysia marked both categories; aothers = blood relations, judicial authority, not sure; ${ }^{\mathrm{b} T a b l e} 3$ provides more information on the types of documentations mentioned by respondents in the survey; 'Figure 3 provides more details on the challenges mentioned by respondents in the survey; ${ }^{d} E D C$ is a computerized system designed for the collection of clinical data in electronic format for use mainly in human clinical trials.

Abbreviations: EC, ethics committee; RA, regulatory authority; EMEA, European Medicines Agency; FDA, US Food and Drug Administration; LAR, legally acceptable representative; PK, pharmacokinetics.

Table 3 List of the documentation/proof required for an individual to be accepted as a legally acceptable representative, according to the survey responses for respondents who indicated "yes" to requirement

\begin{tabular}{llllll}
\hline Country & $\begin{array}{l}\text { Identification } \\
\text { card (parents } \\
\text { and child) }\end{array}$ & $\begin{array}{l}\text { Birth certificate } \\
\text { of child }\end{array}$ & $\begin{array}{l}\text { Marriage } \\
\text { certificate } \\
\text { of parents }\end{array}$ & $\begin{array}{l}\text { Immunization } \\
\text { card of child }\end{array}$ & $\begin{array}{l}\text { Informed consent } \\
\text { form/source notes, } \\
\text { documentation }\end{array}$ \\
\hline $\begin{array}{l}\text { People's Republic of China } \\
\text { Hong Kong }\end{array}$ & $\sqrt{ }$ & $\sqrt{ }$ & $\sqrt{ }$ & $\sqrt{ }$ \\
India & $\sqrt{ }$ & & & $\sqrt{ }$ \\
Indonesia & $\sqrt{ }$ & $\sqrt{ }$ & & \\
Malaysia & $\sqrt{ }$ & $\sqrt{ }$ & $\sqrt{ }$ & \\
Philippines & $\sqrt{ }$ & $\sqrt{ }$ & & \\
Singapore & $\sqrt{ }$ & $\sqrt{ }$ & & \\
Taiwan & & & \\
Thailand & $\sqrt{ }$ & & \\
\hline
\end{tabular}

Notes: *Others: One respondent from the People's Republic of China indicated that the EC has some special requirements. One respondent from Taiwan mentioned "household certificate" as documentation proof.

Abbreviation: EC, ethics committee. 
Table 4 Median pediatric patients (ages 2-19 years) at the sites that responded to the survey

\begin{tabular}{|c|c|c|c|c|c|}
\hline \multirow[t]{2}{*}{ Country } & \multicolumn{5}{|c|}{ Subjects between 2-19 years seen per site per month per therapeutic area (median) } \\
\hline & Respiratory diseases & Oncology & Diabetes & Cardiovascular & Central nervous system \\
\hline Australia & NR & 137 & NR & NR & NR \\
\hline People's Republic of China & 295 & 36 & 19 & 129 & 367 \\
\hline Hong Kong & 164 & 7 & 0 & 0 & 0 \\
\hline India & 128 & 10 & 11 & 14.5 & 41 \\
\hline Indonesia & 15 & NR & NR & NR & 10 \\
\hline Japan & 150 & 42 & 90 & 39 & $|8|$ \\
\hline Malaysia & 74 & 5 & 7 & 12 & 35 \\
\hline New Zealand & 66 & NR & NR & NR & NR \\
\hline Philippines & 60 & 8 & 6 & 14 & 48 \\
\hline Singapore & 33 & 5 & 75 & 72 & 84 \\
\hline South Korea & 590 & 114 & 550 & 30 & 305 \\
\hline Taiwan & 228 & 24 & 173 & 72 & 52 \\
\hline Thailand & 100 & 26 & 12 & 123 & 161 \\
\hline
\end{tabular}

Abbreviation: NR, no response.

been trained to work with infants, children, and their families and when they have routine access to small gauge needles, appropriate topical anesthetics, and even colorful bandages, it helps create the right environment.

Hence, it is important to choose the right sites and investigators with good recruitment histories, the right staff, and appropriate facilities. ${ }^{9}$ A pediatric ophthalmology study, with a $98.5 \%$ retention rate at 3 years' follow-up, conducted a survey with families of the participants. It concluded that expertise in performance of examinations, staff friendliness, and responsiveness, as well as consistency of seeing the same staff at every visit, all played a very important role in patient compliance. ${ }^{26}$

\section{Patient pool}

Understanding the patient referral pathway is key to identifying the most appropriate principal investigator for a clinical trial at a site. Survey responses to the question regarding the primary point of contact for pediatric patients revealed that $93 \%$ of pediatric patients are being managed by pediatricians; the transition to a general practitioner (GP) generally takes place between 12 to 19 years in the Asia Pacific region. This age range at which transition takes place is also reflected in the availability of pediatric patient databases across age groups. Eighty-one percent of the respondents indicated they have a database of patients between $0-10$ years; only $70 \%$ of the respondents indicated they have a database of patients between 11-19 years. This highlights the potential need to consider GPs as subinvestigators in clinical trials for patients above 11 years to ensure good recruitment and compliance.

Table 3 shows the availability of pediatric patients at the sites that responded to the survey. These estimates have several limitations. First, the assessment was based on just one response for a few countries. Second, the findings can only serve as initial reference for understanding the patient availability per country and should be further assessed to understand the actual estimation per protocol. Third, the reason a few countries had no estimation in certain therapeutic areas is due to the specialty of the investigator who responded to the survey; it does not mean that the investigators from the country do not see any patients in the therapeutic area. Even with these limitations, the high level overview is helpful in the initial country selection for a particular protocol. An analysis of pediatric trials listed in ClinicalTrials.gov shows that $23 \%$ are in infectious disease and vaccines, followed by psychiatry and mental illness at $13 \% .{ }^{10}$ Results from the Asia Pacific region show a substantial number of patients in these two therapeutic areas.

As always, in-depth feasibility outreach when a protocol is finalized will provide a better picture of the available patient pool that is under study. Overall, based on the survey results, it would be appropriate to infer that the Asia Pacific countries have a good patient pool for conducting trials in the pediatric population.

\section{Conclusion}

This survey indicates that there is a high level of interest in conducting pediatric trials across various therapeutic indications among sites in the Asia Pacific region. No major insurmountable challenges were identified in conducting pediatric trials. Complexity of the consent and assent process across the countries, as well as the sites, needs to be considered. Also to be considered are the trial-design adaptations for this population to ensure compliance (minimize invasive procedures, placebo control, visit schedules versus school 
timings, etc). There also appears to be an adequate patient pool in many indications, and the availability of a database of subjects can assist in faster screening of subjects for vaccine trials.

\section{Acknowledgments}

The authors would like to thank Quintiles team members across the region that helped reach out to sites in order to complete the survey, particularly Arun Nair, Audrey Ho, Carol Ho, Erika Visser, Henu Tonang, Hitomi Kadowaki, Jane Cai, Kathy Wu, Kenneth Tan, Liling Chen, Maria Rolyn Viaje, Miae Leem, NaRae Baek, Pi-Lien Hung, Piyanuch Tiativiriyakul, Reetiva Dutta, Siew Lee Goh, and Jill Dawson.

\section{Disclosure}

The authors work for Quintiles, a provider of clinical trial services for biopharmaceutical companies worldwide. The authors report no other conflicts of interest in this work.

\section{References}

1. US Food and Drug Administration. The Pediatric Exclusivity Provision, Jan 2001. Status Report to Congress. Silver Spring, MD: US Food and Drug Administration; 2001. Available from: http:// www.fda.gov/downloads/Drugs/DevelopmentApprovalProcess/ DevelopmentResources/UCM049915.pdf. Accessed March 30, 2013.

2. US Congress. Best Pharmaceuticals for Children Act. Washington, DC: US Congress; 2001. Available from: http://www.fda.gov/downloads/ Drugs/DevelopmentApprovalProcess/DevelopmentResources/ UCM049874.pdf. Accessed April 1, 2013.

3. Pediatric Labeling Information Database [database on the Internet]. Silver Spring, MD: US Food and Drug Administration. Available from: http://www.fda.gov/downloads/ScienceResearch/SpecialTopics/ PediatricTherapeuticsResearch/UCM163159.pdf. Accessed April 1, 2013.

4. European Medicines Agency. The European Pediatric Initiative: History of the Paediatric Regulation. London: European Health Organization; July 11, 2007. Available from: http://www.ema.europa.eu/docs/ en_GB/document_library/Other/2009/09/WC500003693.pdf. Accessed April 1, 2013.

5. Bourgeois FT, Murthy S, Pinto C, Olson KL, Ioannidis JP, Mandl KD. Pediatric versus adult drug trials for conditions with high pediatric disease burden. Pediatrics. 2012;130(2):285-292.

6. Smit-Marshall P. Pediatric trials: a worldview. Applied Clinical Trials. 2010;19(1)32-37.

7. Pasquali SK, Burstein DS, Benjamin DK Jr, Smith PB, Li JS. Globalization of pediatric research: analysis of clinical trials completed for pediatric exclusivity. Pediatrics. 2010;126(3):e687-e692.

8. SurveyMethods.com [homepage on the Internet]. Survey software: ask, analyze, improve. SurveyMethods.com; 2012. Available from: https:// www.surveymethods.com/index.aspx. Accessed May 2, 2013.

Pediatric Health, Medicine and Therapeutics

\section{Publish your work in this journal}

Pediatric Health, Medicine and Therapeutics is an international, peerreviewed, open access journal publishing original research, reports, editorials, reviews and commentaries. All aspects of health maintenance, preventative measures and disease treatment interventions are addressed within the journal. Practitioners from all disciplines are invited to submit
9. World Health Organization. Children are Not Little Adults. Geneva: World Health Organization; Jul 2008. Available from: http://www.who.int/ceh/ capacity/Children_are_not_little_adults.pdf. Accessed April 1, 2013.

10. Committee on Clinical Research Involving Children. The Ethical Conduct of Clinical Research Involving Children. Washington, DC: Institute of Medicine of the National Academies; 2004. Available from: http://www.ncbi. nlm.nih.gov/books/NBK25557/pdf/TOC.pdf. Accessed April 1, 2013.

11. Pasquali SK, Lam WK, Chiswell K, Kemper AR, Li JS. Status of the pediatric clinical trials enterprise: an analysis of the US ClinicalTrials. gov registry. Pediatrics. 2012;130(5):e1269-e1277.

12. ICH.org [homepage on the Internet]. Guidelines for clinical investigation of medicinal products in the pediatric population (E11). International Conference on Harmonisation; 2000 [cited 2001]. Available from: http://www.ich.org/products/guidelines/efficacy/article/efficacyguidelines.html. Accessed April 1, 2013.

13. Flynn JT. Ethics of placebo use in pediatric clinical trials: the case of antihypertensive drug studies. Hypertension. 2003;42(5):865-869.

14. Gómez-Díaz RA, Rodarte NW, Robles SC, Aguilar-Salinas CA, Jonas SD, Güinzberg AL. The ethical use of placebo in pediatric research. Journal of Clinical Research and Bioethics. Available from: http://www.omicsonline.org/2155-9627/2155-9627-2-120.php. Accessed Apr 1, 2013.

15. Smith PB, Li JS, Murphy MD, Califf RM, Benjamin DK Jr. Safety of placebo controls in pediatric hypertension trials. Hypertension. 2008;51(4):829-833.

16. Jadhav M, Bhath A. Ethics in clinical research in India: a survey of clinical research professionals' perceptions. Perspect Clin Res. 2013;4(1):4-8.

17. Meibohm B, Läer S, Panetta J, Barrett J. Population pharmacokinetic studies in pediatrics: issues in design and analysis. AAPS $J$. 2005;7(2):E475-E487.

18. Gross T. Challenges and practicalities of obtaining parental consent and child assent in pediatric trials. Regulatory Rapporteur. 2010;7(6):15-18.

19. Whittle A, Shah S, Wilfond B, Gensler G, Wendler D. Institutional review board practices regarding assent in pediatric research. Pediatrics. 2004;113(6):1747-1752.

20. American Academy of Pediatrics Committee on Drugs. Guidelines for the ethical conduct of studies to evaluate drugs in pediatric populations. Pediatrics. 1995;95(2)286-294.

21. Hays J. FactsandDetails.com [homepage on the Internet]. One child policy in China. FactsandDetails.com. Available from: http:// factsanddetails.com/china.php?itemid=128. Accessed May 1, 2013.

22. Barfield RC, Church C. Informed consent in pediatric clinical trials. Curr Opin Pediatr. 2005;17(1):20-24.

23. Parekh S. Pediatric consent errors. Applied Clinical Trials. Available from: http://www.appliedclinicaltrialsonline.com/appliedclinicaltrials/ article/articleDetail.jsp?id=738744. Accessed May 1, 2013.

24. Clay DL. Commentary: rethinking our interventions in pediatric chronic pain and treatment research. J Pediatr Psychol. 2000;25(1):53-55.

25. Rieder MJ. Conducting global pediatric clinical trials. PharmaFocusAsia. com. 2013. Available from: http://www.pharmafocusasia.com/ knowledge_bank/articles/paediatric_clinicaltrials.htm. Accessed April 2, 2013.

26. Dias L, Schoenfeld E, Thomas J, et al. Reasons for high retention in pediatric clinical trials: comparison of participant and staff responses in the Correction of Myopia Evaluation Trial. Clin Trials. 2005;2(5): 443-452.

\section{Dovepress}

their work as well as healthcare researchers and patient support groups. The manuscript management system is completely online and includes a very quick and fair peer-review system. Visit http://www.dovepress.com/ testimonials.php to read real quotes from published authors. 